\title{
Oxidative stress in older adults: effects of physical fitness
}

\author{
Tinna Traustadóttir • Sean S. Davies • Yali Su • \\ Leena Choi • Holly M. Brown-Borg • \\ L. Jackson Roberts II • S. Mitchell Harman
}

Received: 29 March 2011 / Accepted: 30 May 2011 / Published online: 14 June 2011

(C) American Aging Association 2011

\begin{abstract}
Acute exercise results in transient change in redox balance. High concentrations of reactive oxygen species (ROS) can lead to oxidative damage to macromolecules. However, moderate periodic increases in ROS, such as experienced with habitual exercise, may activate signal transduction pathways which stimulate
\end{abstract}

Electronic supplementary material The online version of this article (doi:10.1007/s11357-011-9277-6) contains

supplementary material, which is available to authorized users.

T. Traustadóttir $(\bowtie) \cdot$ S. M. Harman

Kronos Longevity Research Institute,

2222 E. Highland Ave., Suite 220,

Phoenix, AZ 85016, USA

e-mail: tinna.traustadottir@kronosinstitute.org

S. S. Davies $\cdot$ L. J. Roberts II

Department of Pharmacology,

Vanderbilt University School of Medicine,

Nashville, TN, USA

Y. Su

Kronos Science Laboratory,

Phoenix, AZ, USA

L. Choi

Department of Biostatistics,

Vanderbilt University School of Medicine,

Nashville, TN, USA

H. M. Brown-Borg

Department of Pharmacology, Physiology,

and Therapeutics, University of North Dakota School

of Medicine and Health Sciences,

Grand Forks, ND, USA increases in endogenous antioxidant systems. This study tested the hypothesis that physically fit older adults would have less oxidative stress than unfit age-matched controls, due to greater circulating concentrations of non-enzymatic antioxidants and greater capacity to upregulate antioxidant enzymes. We compared 37 fit (mean age 65.2 \pm 5 years) and 35 unfit (mean age 67.7 \pm 4 years) men and women. Fitness status was classified by $\mathrm{VO}_{2}$ max and maximal leg power. Basal levels of oxidative stress were assessed by measuring urinary markers of nucleic acid damage and lipid peroxidation. Antioxidant status was assessed by measuring total antioxidant power and ratios of reduced to oxidized glutathione in plasma, at rest. The capacity to counteract an oxidative insult was assessed by measuring changes in plasma $\mathrm{F}_{2}$-isoprostanes in response to forearm ischemia-reperfusion. The fit individuals had significantly lower levels of urinary markers of oxidative damage (all $P<0.05$ ) and lower $\mathrm{F}_{2}$-isoprostane response to the oxidative challenge $(P<0.05)$, but there were no group differences in antioxidant status. The lower levels of oxidative stress in the fit individuals were not mediated by known effects of exercise training such as adiposity, HDL concentrations, or small molecular weight antioxidants. These data suggest that reduced oxidative stress associated with physical fitness results from differences in activity of antioxidant enzymes.

Keywords $\mathrm{F}_{2}$-isoprostanes · Ischemia-reperfusion . Exercise · Glutathione · 8-Hydroxy-2'-deoxyguanosine 


\section{Introduction}

Transient increases of reactive oxygen species (ROS) are essential for normal cell signaling, but high concentrations of ROS also lead to oxidative modification of macromolecules and tissue damage. Small molecular weight antioxidants and antioxidant enzymes present in tissue can limit this collateral oxidative damage by eliminating ROS and thus rapidly resetting the redox tone to resting levels. Transient increases in ROS also upregulate endogenous antioxidant enzymes so that regular exposure to mild transient increases in ROS may ultimately increase an organism's capacity to limit oxidative damage. Conversely, aging is associated with a decrease in the ability to limit and repair oxidative damage and ensuing oxidative damage is thought to be a key mechanism in the aging process (Harman 1956; Sohal and Weindruch 1996; Ashok and Ali 1999). Oxidative modifications of macromolecules have been implicated in many pathological processes including atherosclerosis, cancer, diabetes, and Alzheimer's disease (Butterfield et al. 1999; Spector 2000).

Exercise training can ameliorate or counteract many of the physiological decrements of aging and is associated with decreased risk for many of the diseases linked to excessive oxidative stress (Paffenbarger et al. 1993; Church et al. 2002; LaMonte et al. 2005). One potential mechanism for this increased resistance to age-related diseases may be that physical activity induces adaptations that increase resistance to oxidative damage. Data from animal studies show that regular exercise training can prevent the age-associated increase in membrane lipid peroxidation in several tissues (Radak et al. 2004; Rosa et al. 2005). Results of studies on effects of exercise training on oxidative stress in humans have been more equivocal with some studies reporting significant protective effects (Vincent et al. 2006; Knez et al. 2007) and others no protective effect (Marzatico et al. 1997; Santos-Silva et al. 2001; Pittaluga et al. 2006). Differences in biomarker selection (e.g., $\mathrm{F}_{2}$-isoprostanes versus thiobarbituric acid reactive substances) and measurement method (e.g., mass spectrometry versus enzyme-linked immunosorbent assays) could explain differences in study results. Another possible reason for this inconsistency is the greater heterogeneity among human populations compared to studies with inbred rodents in highly controlled environments. Differences in genetic back- ground, diet, and environmental exposures contribute to variations in an individual's resting levels of oxidative stress and may therefore mask the effects of exercise training on oxidative damage. Significant changes in antioxidant capacity due to exercise training might be more easily detected by examining the effect of exercise on response to an acute oxidative challenge, which provides a more robust test of an individual's antioxidant capacity.

Previous studies that have measured oxidative stress responses under challenge conditions have generally used acute exercise as a physiological challenge (Khassaf et al. 2001; Miyazaki et al. 2001; Sacheck et al. 2003; Fatouros et al. 2004; Choi and Cho 2007; Magalhaes et al. 2007; Nikolaidis et al. 2007; Simar et al. 2007; Falone et al. 2010). However, using an acute exercise challenge to induce ROS complicates the interpretation of the effect of exercise training and physical fitness on oxidative stress responses because exercise training generally increases an individual's tolerance of exercise load including total metabolic capacity $\left(\mathrm{VO}_{2} \max \right)$ and the relative workload that elicits lactate accumulation (anaerobic threshold). Because a greater absolute workload is generally required to elicit similar physiological responses and metabolic stress to acute exercise in trained individuals, a finding of less oxidative stress in trained versus untrained individuals at the same absolute workload may simply represent a relative workload below that required to induce oxidative stress. On the other hand, failure to find less oxidative stress in trained individuals tested at the same relative exercise workload $\left(\% \mathrm{VO}_{2} \max \right)$ cannot be unambiguously interpreted to indicate that trained individuals in fact have similar resistance to oxidative stress as untrained individuals because greater resistance to oxidative stress may contribute to their ability to sustain a greater absolute workload. Therefore, a non-exercise-related inducer of oxidative stress should be a more straightforward test of whether exercise training and consistent physical activity lead to systemic changes in the capacity to resist oxidative stress. For this reason, we have used forearm ischemia-reperfusion $(\mathrm{I} / \mathrm{R})$ induced by inflation of a blood pressure cuff as a non-exercise-related, acute oxidative challenge. Forearm $\mathrm{I} / \mathrm{R}$ is an effective method to temporarily increase ROS, allowing us to quantify the response and recovery by measuring changes in plasma levels of $\mathrm{F}_{2}$-isoprostanes, a marker 
of lipid peroxidation (Davies et al. 2009; Traustadóttir et al. 2009). Using this method, we have previously shown that older adults have a diminished capacity to constrain an acute oxidative challenge as compared to young adults (Davies et al. 2009). Additionally, we have shown that the capacity to constrain this oxidative challenge is increased after a 2-week intervention with tart cherry juice, a natural dietary antioxidant (Traustadóttir et al. 2009).

We hypothesized that older individuals with high physical fitness due to exercise training would have a greater capacity to limit oxidative damage than their unfit counterparts, and that this increased capacity might be due both to greater circulating concentrations of small molecular weight antioxidants and greater capacity to upregulate antioxidant enzymes. To test this hypothesis, we measured levels of oxidative damage and antioxidant status under resting conditions as well as $\mathrm{F}_{2}$-isoprostane responses to forearm $\mathrm{I} / \mathrm{R}$ in fit and unfit healthy older adults (60-78 years). The primary aim of this study was to ascertain whether physically fit older adults would exhibit lower levels of oxidative damage under resting conditions and in response to the forearm I/R, compared to healthy age-matched controls with lower physical fitness. Systemic oxidative damage was assessed by measuring urinary markers of nucleic acid damage and lipid peroxidation. Antioxidant status was assessed by measuring the reduced to oxidized glutathione ratio (GSH/GSSG) and total antioxidant power (TAP).

\section{Materials and methods}

\section{Subjects}

Study participants were recruited by sending letters to individuals in Kronos Longevity Research Institute (KLRI) database as well as commercially available databases, advertisements in newsletters, and by word of mouth. We aimed to recruit 40 fit and 40 unfit participants, 60-80 years of age, equally divided between men and women. All participants were in general good health by self-report, did not smoke, and were not overly obese $(\mathrm{BMI} \leq 32)$. Exclusion criteria included history of chronic disease, estrogen or testosterone supplementation within the previous 6 months, and any condition that would contraindicate maximal exercise testing as outlined by the
American College of Sports Medicine (American College of Sports et al. 2005) such as evidence of previous myocardial infarction on EKG, clinically significant arrhythmia on a resting EKG or significant EKG changes during the maximal oxygen consumption $\left(\mathrm{VO}_{2}\right.$ max $)$ test, elevated resting blood pressure, or musculoskeletal problems. Additionally, we excluded persons taking antioxidant supplementation in excess of the equivalent of a standard multi-vitamin. The protocol was approved by and performed under the guidelines of the Western Institutional Review Board, and all participants provided written informed consent prior to entering the study.

A total of 108 individuals signed informed consent and were pre-screened. Of those, nine were excluded from study participation due to conditions listed as exclusion criteria for the study [BMI too high $(n=2)$, hypertension $(n=2)$, evidence of left bundle branch block on resting EKG $(n=2)$, evidence of coronary artery disease on resting EKG, evidence of chronic disease $(n=2)$ ]. Exercise testing was completed on 99 individuals and of those 19 were excluded from study participation [positive stress test $(n=5)$, did not meet $\mathrm{VO}_{2}$ max and/or leg power criteria $\left.(n=14)\right]$. Three participants dropped out of the study; two after completing the two exercise testing sessions citing time limitations and concerns with blood draws and one participant due to spouse's concern with the safety of the I/R test. Additionally, after analysis of resting plasma $\mathrm{F}_{2}$-isoprostane levels, we found that five individuals (two fit, three unfit) had resting plasma $\mathrm{F}_{2}$-isoprostane levels that were greater than 2 standard deviations from the mean $\left(>80 \mathrm{pg} \mathrm{ml}^{-1}\right.$, see Supplemental Figure 1). Because these values differed so significantly from the general group, and potentially represented individuals under elevated oxidative stress for reasons unrelated to physical fitness, these individuals were excluded from the main data analysis. Thus, 72 individuals were included in the main data analyses; 37 fit (18 men and 19 women) and 35 unfit (18 men and 17 women). Inclusion of the resting $\mathrm{F}_{2}$-isoprostanes identified as outliers did not alter overall differences between the fit and unfit group, but did increase group variance. Analysis of data with these individuals included 39 fit (19 men and 20 women) and 38 unfit (19 men and 19 women) and is provided in the supplemental data for comparison (Supplemental Figure 2 and Supplemental Table 1). 


\section{Study design}

The study was a cross-sectional comparison between fit and unfit older men and women. Group designation, i.e., fitness classification, was made based on measurements of maximal oxygen consumption $\left(\mathrm{VO}_{2}\right.$ max $)$ and maximal leg power (see descriptions below) using a priori specific criteria adjusted for age (60-69 years, 70-80 years) and gender (see Table 1). The $\mathrm{VO}_{2}$ max criteria were derived from fitness classification criteria published by the American Heart Association, and data from the literature, adjusted for cycle ergometry versus treadmill testing (AHA 1972; Spina et al. 2000; Stathokostas et al. 2004; Traustadóttir et al. 2004). The leg power criteria were derived from published and unpublished data from our laboratory on leg power testing in older men and women, as well as personal communication with researchers doing these same measurements (de Vos et al. 2005; Traustadóttir et al. 2008). Participants completed a pre-screening visit prior to the exercise testing, which included a short physical exam, measurements of height, weight, resting blood pressure, resting $\mathrm{EKG}$, and a fasted blood draw for standard $\mathrm{CBC}$ and chemistry panel. In order to qualify for the study, individuals had to be above (fit) or below (unfit) the specific criteria for both $\mathrm{VO}_{2}$ max and maximal leg power shown in Table 1. To account for a possible learning effect and to increase the accuracy of the fitness classification, the exercise testing was repeated at a visit subsequent to the primary screening visit. Prior to the last study visit at which the participants underwent the forearm ischemia-reperfusion trial, they were asked to collect urine samples on five separate days and bring the frozen samples to the laboratory on

Table 1 Criteria for fit and unfit in men and women

\begin{tabular}{lllll}
\hline Sex & Age & Category & $\begin{array}{l}\mathrm{VO}_{2} \mathrm{max}^{-1} \\
\left(\mathrm{ml} \mathrm{kg}^{-1} \mathrm{~min}^{-1}\right)\end{array}$ & $\begin{array}{l}\text { Leg power } \\
\left(\mathrm{W} \mathrm{kg}^{-1}\right)\end{array}$ \\
\hline \multirow{2}{*}{ Men } & $60-69$ & Fit & $\geq 31$ & $>15.5$ \\
& & Unfit & $\leq 26$ & $\leq 15.5$ \\
& $70-80$ & Fit & $\geq 28$ & $>14.5$ \\
& & Unfit & $\leq 23$ & $\leq 14.5$ \\
Women & $60-69$ & Fit & $\geq 24$ & $>11.0$ \\
& & Unfit & $\leq 20$ & $\leq 11.0$ \\
& \multirow{2}{*}{$70-80$} & Fit & $\geq 21$ & $>9.5$ \\
& & Unfit & $\leq 18$ & $\leq 9.5$ \\
& & & &
\end{tabular}

the day of the visit. A diagram of the study visits is shown in Fig. 1.

Measures of physical fitness

Maximal oxygen consumption $\left(\mathrm{VO}_{2}\right.$ max $)$ was measured with a graded exercise test performed on a cycle ergometer as previously described (Traustadóttir et al. 2008). Briefly, oxygen uptake was measured via indirect calorimetry using an integrated metabolic measurement cart (SensorMedics Vmax, Yorba Linda, CA, USA). Heart function was monitored with continuous 12-lead EKG, and blood pressure was measured every $3 \mathrm{~min}$. $\mathrm{VO}_{2} \max$ was considered achieved if two of the following three criteria were met: (1) a plateau in $\mathrm{VO}_{2}$ with an increase in workload, (2) a respiratory exchange ratio (RER) $\geq 1.10$, or (3) heart rate within 10 beats of the age-predicted maximal heart rate calculated as $220 \mathrm{bpm}$-age (Kohrt et al. 1991). Standard contraindications to exercise testing, as well as termination criteria, outlined by the American College of Sports Medicine (2005) were followed.

Peak leg power was determined using a Keiser pneumatic seated leg press fitted with a Keiser A420 electronic package (Keiser Corp., Fresno, CA, USA) as previously described (Traustadóttir et al. 2008). This equipment allows determination of force, velocity, and power output per repetition performed and automatically downloads these data to a personal computer. After assessing maximal strength via a standard one-repetition maximum (1-RM) test, power output was determined at $50 \%, 60 \%$, and $70 \%$ of 1 RM. Subjects were instructed to complete the concentric portion of the exercise movement as rapidly as possible. Participants completed five individual trials at each workload with $30 \mathrm{~s}$ of rest between each attempt. The highest power achieved (using all three workloads) was used to classify the individual's fitness level.

\section{Lifetime physical activity history}

The objective measures of physical fitness $\left(\mathrm{VO}_{2}\right.$ max and leg power) mainly represent the individual's status at the time of testing. We were also interested in how physically active the study participants had been in general over the course of their lifetime. Since oxidative damage is thought to accumulate with age and exercise 
Fig. 1 Schematic of study visits
Telephone screening for study eligibility

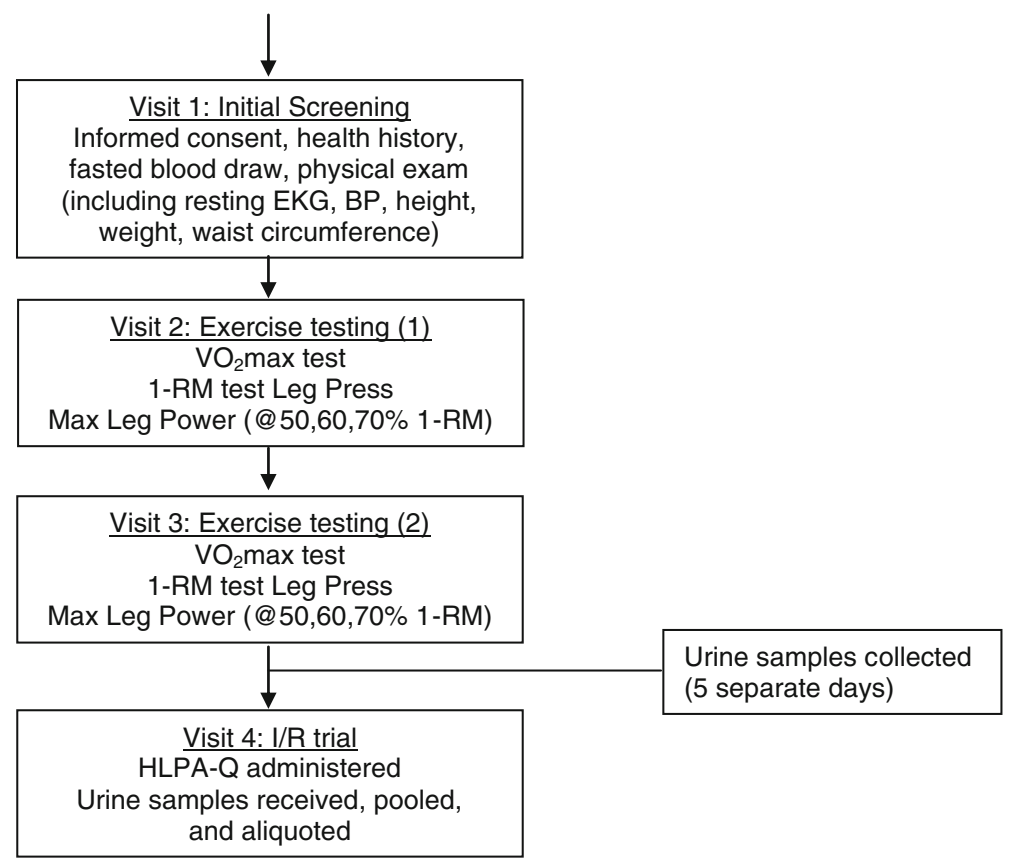

is believed to protect from oxidative damage, the amount of physical activity over one's lifetime might also affect oxidative stress status with aging. Lifetime physical activity history was assessed using a modified version (Cottreau et al. 2000) of the Historical Leisure Physical Activity Questionnaire by Kriska (1997). Subjects were interviewed and asked to rank the frequency of the physical activities they performed during the following periods: ages $14-17,18-21,22-$ $29,30-39,40-49,50-59,60-69$, and $>70$ years on a scale of $0-4(0=$ never; $1=<1 \mathrm{~h} /$ week; $2=1-3 \mathrm{~h} /$ week, $3=4-6 \mathrm{~h} /$ week; $4=>6 \mathrm{~h} /$ week $)$. The reliability and validity of this questionnaire have been previously established (Kriska et al. 1991). A lifetime leisure physical activity index (HLPA score) was calculated by averaging activity level rankings (range $0-4$ ) across all ages in each person's life to provide a single estimate of lifetime physical activity. HLPA score $>3$ was categorized as "high" lifetime physical activity, a score of 2-3 was categorized as "moderate", and a score $<2$ was considered "low" lifetime physical activity.

Forearm ischemia-reperfusion (I/R) trial

Study participants reported to the KLRI Clinical Research Center in the morning and completed the $\mathrm{I} / \mathrm{R}$ trial as previously described (Davies et al. 2009;
Traustadóttir et al. 2009). Study participants were instructed to consume a light breakfast without caffeinated beverages. Upon arrival, an intravenous catheter was inserted in the non-dominant arm and a baseline blood sample was collected $(-34 \mathrm{~min})$. The catheter was kept in situ with a slow saline drip throughout the trial. A blood pressure cuff was placed on the same arm, inflated to $200 \mathrm{mmHg}$ and kept inflated for $10 \mathrm{~min}$, then released for $2 \mathrm{~min}$. This inflation procedure was repeated twice more (total time $=34 \mathrm{~min}$ ). At the three time points of cuff inflation when blood flow was occluded, approximately $2 \mathrm{ml}$ of heparin flush (10 kUSP/l) was injected into the blood sample tubing to prevent any clotting at the site. A blood sample was drawn immediately after the final release of the blood pressure cuff and then at minutes $15,30,60,120,180$, and 240 postfinal cuff deflation.

The blood samples were centrifuged and the plasma layer extracted and frozen at $-80^{\circ} \mathrm{C}$ until analysis. Free $\mathrm{F}_{2}$-isoprostanes in plasma were quantified, after purification and derivatization, using gas chromatography/negative ion chemical ionizationmass spectrometry with $\left[{ }^{2} \mathrm{H}_{4}\right] 15-\mathrm{F}_{2 \mathrm{t}}$-isoP as an internal standard (Morrow and Roberts 1999). Compounds were analyzed as pentafluorobenzyl ester, trimethylsilyl ether derivatives by monitoring mass- 
to-charge ratios of 569 and 573 for endogenous $\mathrm{F}_{2}$ isoprostanes and the $\left[{ }^{2} \mathrm{H}_{4}\right] 15-\mathrm{F}_{2 \mathrm{t}}$-isoP internal standard, respectively.

Resting (non-challenged) oxidative stress levels

To assess oxidative damage under resting (nonchallenged) conditions, urinary excretion of oxidatively modified nucleic acids and lipids was measured. Study participants collected a first-morning voided urine sample on five separate days. They were asked to refrain from any vigorous exertion on the day preceding the sampling. The five individual urine samples were pooled by mixing an equal amount from each daily sample. The pooled sample was then aliquoted and stored at $-20^{\circ} \mathrm{C}$ until analyses. Urinary creatinine was measured on a Sirrus Clinical Chemistry Analyzer (Stanbio Laboratory, Boerne, TX, USA) using standard clinical methodology. Measurement of urinary $\mathrm{F}_{2}$-isoprostanes, 8-hydroxy-2'-deoxyguanosine (8$\mathrm{OHdG})$, and 8-hydroxyguanosine (8-oxo-G) was performed using a liquid chromatographic-tandem mass spectrometry as previously described (Harman et al. 2003; Liang et al. 2003). As an index of systemic lipid peroxidation, we calculated the sum of four isomers of isoprostanes $\left(15-\mathrm{F}_{2 \mathrm{t}}-\mathrm{IsoP}, 5-\mathrm{F}_{2 \mathrm{t}}\right.$-IsoP, 5- $\mathrm{F}_{2 \mathrm{c}}$-IsoP, and 2,3-dinor $15-\mathrm{F}_{2 \mathrm{t}}$-isoP) in the urine samples. We analyzed 8-hydroxy-2'-deoxyguanosine (8-OHdG) and 8-hydroxyguanosine (8-oxo-G) separately as markers of DNA and of RNA damage, respectively. Both lipid and nucleic acid markers were adjusted for urinary dilution effects by expressing them as micrograms per gram of creatinine.

\section{Antioxidant status}

Glutathione To assess antioxidant status, glutathione was measured in whole blood, taken at the baseline sampling of the $\mathrm{I} / \mathrm{R}$ trial. The blood sample was drawn into an EDTA $\left(\mathrm{K}_{3}\right)$ tube and immediately placed on ice for $5 \mathrm{~min}$. Whole blood was aliquoted and mixed with 5\% metaphosphoric acid solution, incubated on ice for $5 \mathrm{~min}$, and then centrifuged. The supernatant was transferred to a microcentrifuge tube and stored at $-80^{\circ} \mathrm{C}$ until analysis. Protein concentration was determined in each sample using a Bradford assay (Bradford 1976). One hundred microliters of picric acid was added to $100 \mu \mathrm{l}$ of plasma and vortexed for $15 \mathrm{~s}$. These samples were then centri- fuged for $3 \mathrm{~min}$ at 10,000 rpm. The cellular oxidation state was determined in the supernatant fractions by measuring the ratio of a specific reduction/oxidation pair, GSH/glutathione disulfide (GSSG) using standard recycling methods described by Griffith (1986) and employed in our laboratory as previously described (Brown-Borg et al. 1999). The GSH content was determined by using a standard curve generated from known concentrations of glutathione. One half of each sample was used for GSSG determination and the other half for GSH. Samples for GSSG determination were incubated at room temperature with 4-vinyl pyridine (4-VP) for $1 \mathrm{~h}$. The GSSG (as GSHx2) is then subtracted from the total GSH to determine actual GSH level.

Total antioxidant power (TAP) The capacity of plasma to reduce $\mathrm{Cu}^{+2}$ to $\mathrm{Cu}^{+1}$ is a direct measure of its antioxidant capacity and was measured using a commercial total antioxidant power assay (Oxford Biochemical Research, Oxford, MI, USA) with uric acid as an external standard. All results are expressed as millimolar uric acid equivalents.

Statistical analyses

Group differences in subject characteristics were analyzed with two-sample $t$ tests. Linear regression analyses with cluster bootstrap methods were used to test whether $\mathrm{F}_{2}$-isoprostanes levels differed between fit and unfit in response to the $\mathrm{I} / \mathrm{R}$ trial. Cluster bootstrap methods (1,000 bootstraps) were used to take into account correlation within subject due to the repeated measurements. The $\mathrm{F}_{2}$-isoprostane levels at baseline and $\mathrm{F}_{2}$-isoprostane levels were logarithmic transformed to reduce skewness of the distributions. The models included time and quadratic time trend to adjust for nonlinear time trend. Potential confounding factors such as the $\mathrm{F}_{2}$-isoprostane level at baseline, age, gender, and BMI were adjusted. Analyses were performed using STATA 11.0 (StataCorp, College Station, TX, USA). The integrated $\mathrm{F}_{2}$-isoprostane response for each individual was calculated as the area-under-the-response-curve (AURC) by the method of the trapezoidal rule. A univariate GLM analyses was used for comparisons between groups for the mean AURC responses, the GSH/GSSG ratio, TAP, and the urinary markers of oxidative 
stress, adjusting for covariates. Pearson correlation and linear regression analyses were used to examine the relationship between various outcome variables. These analyses were conducted using PASW Statistics 18.0 software (SPSS, Chicago, IL, USA). All comparison was considered significant at $P<0.05$. Data are presented as means \pm SEM except for Table 2, in which the data are presented as means \pm SD.

\section{Results}

Subject characteristics are detailed in Table 2. As expected, the fit group had significantly higher $\mathrm{VO}_{2}$ max and maximal leg power as compared to the unfit group $(P<0.001)$.

Table 2 Subject characteristics and laboratory measures

\begin{tabular}{|c|c|c|}
\hline Variable & Unfit $(n=35)$ & Fit $(n=37)$ \\
\hline \multicolumn{3}{|l|}{ Subject characteristics } \\
\hline Age (years) & $67.7 \pm 4.3$ & $65.2 \pm 4.7^{*}$ \\
\hline Height $(\mathrm{cm})$ & $168 \pm 11$ & $170 \pm 10$ \\
\hline Weight (kg) & $78.2 \pm 17.1$ & $71.6 \pm 14.4$ \\
\hline BMI $\left(\mathrm{kg} / \mathrm{m}^{2}\right)$ & $27.3 \pm 3.7$ & $24.5 \pm 3.0^{* *}$ \\
\hline $\mathrm{WC}(\mathrm{cm})$ & $94 \pm 11$ & $85 \pm 12 * *$ \\
\hline SBP $(\mathrm{mmHg})$ & $124 \pm 13$ & $120 \pm 11$ \\
\hline $\mathrm{DBP}(\mathrm{mmHg})$ & $78 \pm 7$ & $75 \pm 8$ \\
\hline $\mathrm{VO}_{2} \max \left(\mathrm{ml} \mathrm{kg}^{-1} \min ^{-1}\right)$ & $18.6 \pm 3.1$ & $30.0 \pm 6.7 * * *$ \\
\hline Maximal leg power $\left(\mathrm{W} \mathrm{kg}^{-1}\right)$ & $11.9 \pm 2.7$ & $16.8 \pm 4.5^{* * *}$ \\
\hline HLPA score & $2.3 \pm 1.0$ & $3.4 \pm 0.5 * * *$ \\
\hline \multicolumn{3}{|l|}{ Laboratory measures } \\
\hline Hematocrit (\%) & $42.4 \pm 3.5$ & $42.0 \pm 3.4$ \\
\hline Total cholesterol (mg/dl) & $192 \pm 31$ & $215 \pm 38 * *$ \\
\hline Creatinine, serum (mg/dl) & $1.0 \pm 0.3$ & $1.0 \pm 0.3$ \\
\hline Glucose, fasting (mg/dl) & $100 \pm 14$ & $96 \pm 9$ \\
\hline HDL-C (mg/dl) & $52 \pm 16$ & $65 \pm 17 * *$ \\
\hline LDL-C (mg/dl) & $124 \pm 30$ & $139 \pm 38$ \\
\hline Iron $(\mu \mathrm{g} / \mathrm{dl})$ & $99 \pm 41$ & $101 \pm 26$ \\
\hline Triglycerides (mg/dl) & $126 \pm 90$ & $95 \pm 57$ \\
\hline Uric acid (mg/dl) & $4.5 \pm 1.7$ & $4.2 \pm 1.3$ \\
\hline
\end{tabular}

Values are means \pm SD

$B M I$ body mass index, $W C$ waist circumference, $\mathrm{VO}_{2}$ max maximal oxygen consumption, HLPA historical leisure physical activity

$* P<0.05, * * P<0.01, * * * P<0.001$
Additionally, the fit group reported high lifetime physical activity (HLPA $>3.0)$ while the unfit group reported moderate lifetime physical activity (HLPA= 2.0-3.0), and the group differences in mean HLPA scores were statistically significant $(P<0.001)$. The mean age was slightly lower in the fit group and they had lower BMI and smaller waist circumference. Routine laboratory values did not differ between the groups except that the fit group had significantly higher HDL as well as total cholesterol levels. Analyses were adjusted for age, BMI, HDL cholesterol, and gender, when appropriate.

Effects of fitness on baseline levels of oxidative stress biomarkers

To assess whether increased physical fitness reduced baseline levels of oxidative stress, we measured plasma levels of $F_{2}$-isoprostanes and urinary levels of $\mathrm{F}_{2^{-}}$ isoprostanes and oxidized nucleic acids. Although plasma $\mathrm{F}_{2}$-isoprostane levels were somewhat lower in fit individuals, these differences did not reach statistical significance $\left(34.2 \pm 1.9\right.$ vs. $37.9 \pm 2.6 \mathrm{pg} \mathrm{m}^{-1}$ in fit and unfit, respectively, $P=0.254$ ). The fit group had significantly lower levels of urinary $F_{2}$-isoprostanes $\left(5.28 \pm 0.31\right.$ vs. $5.59 \pm 0.32 \mu \mathrm{g} \mathrm{g}^{-1}$ creatinine, fit and unfit, respectively; $P=0.002$, Fig. 2a). The fit group also had significantly lower levels of markers of nucleic acid damage: 8-hydroxy-2'-deoxyguanosine (8-OHdG), a marker of DNA damage $(5.19 \pm 0.22$ vs. $5.91 \pm 0.24 \mu \mathrm{g} \mathrm{g}^{-1}$ creatinine, fit and unfit, respectively; $P=0.02$, Fig. 2b), and 8-hydroxyguanosine (8-oxo-G), a marker of RNA damage $(5.91 \pm 0.21$ vs. $7.05 \pm$ $0.32 \mu \mathrm{g} \mathrm{g}^{-1}$ creatinine, fit and unfit, respectively; $P=0.001$, Fig. 2c).

Although current physical fitness (i.e., $\mathrm{VO}_{2} \max$ ) correlates to current physical activity, the fit individuals also had higher historical leisure physical activity (HLPA) so that adaptations that lower oxidative stress might be acquired during earlier life stages or as a result of cumulative physical activity over the lifetime of the individual. To determine if current oxidative stress status could be predicted by HLPA, we compared baseline levels of oxidative stress markers with self-reported HLPA. We found that increased HLPA negatively correlated with urinary levels of oxidatively modified nucleic acids (Table 3 ), but not $\mathrm{F}_{2}$-isoprostanes, suggesting that HLPA may contribute to decreased oxidative stress. Further studies in 

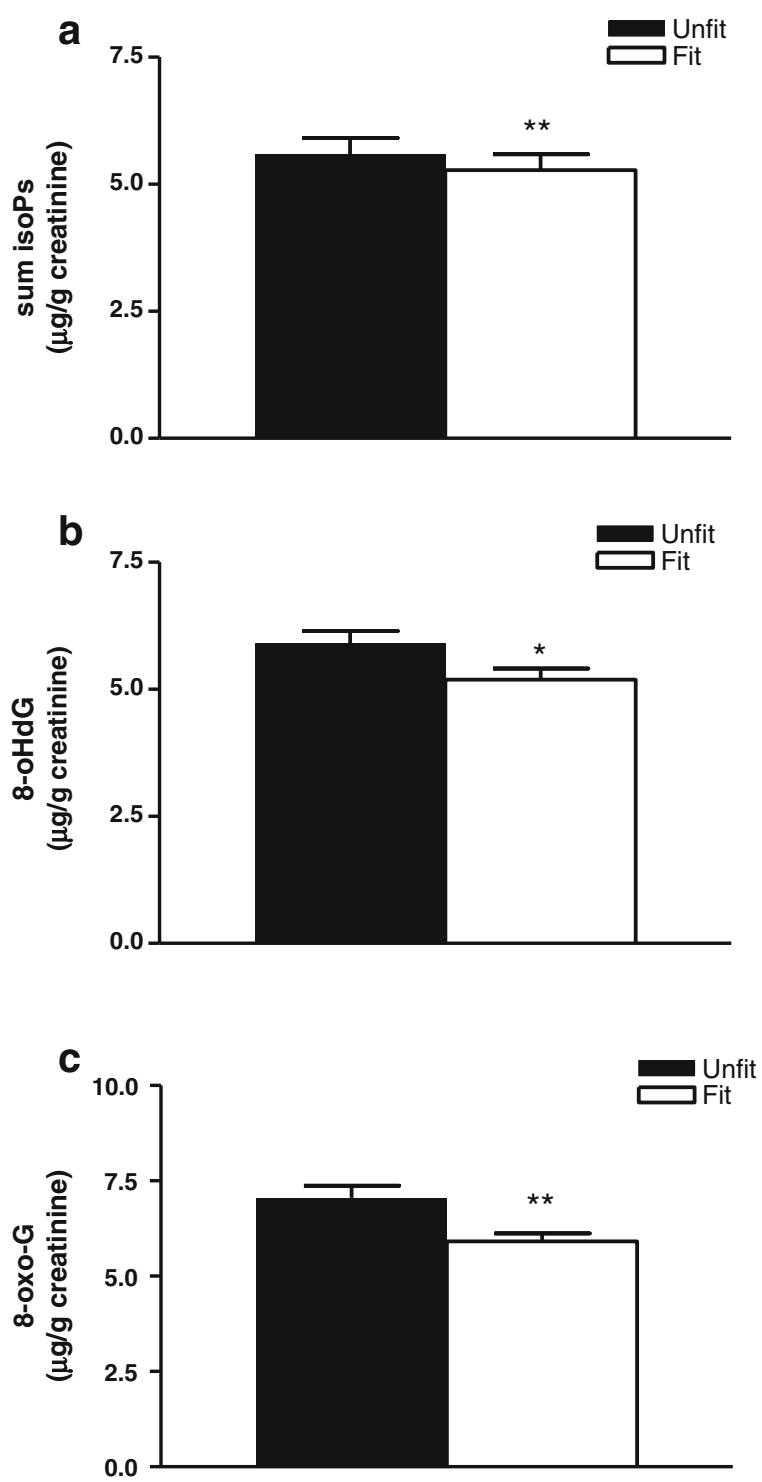

Fig. 2 Urinary markers of oxidative damage in unfit (black bar) and fit (white bar) older adults. Values are means \pm SEM. The fit group had significantly lower levels of all three markers as designated: ${ }^{*} P<0.05,{ }^{*} * P<0.01$. a $\mathrm{F}_{2}$-isoprostanes (sum of four isomers- $15-\mathrm{F}_{2 \mathrm{t}}$-IsoP, $5-\mathrm{F}_{2 \mathrm{t}}-\mathrm{IsoP}, 5-\mathrm{F}_{2 \mathrm{c}}$-IsoP, and 2,3-dinor $15-\mathrm{F}_{2 \mathrm{t}}$-isoP). b 8 -Hydroxy-2'-deoxyguanosine (8-OHdG). c 8 -Hydroxyguanosine (8-oxo-G)

subjects with high HLPA, with or without currently high $\mathrm{VO}_{2}$ max, will be needed to determine the specific contributions of HLPA and current physical fitness to reduced baseline oxidative stress.

Increased physical fitness significantly affects several parameters that may contribute to antioxidant capacity including increased HDL-C levels and reduced visceral adiposity. To determine whether changes in these parameters might account for effects of increased physical fitness on baseline levels of oxidative stress markers, we also analyzed the correlations between these parameters (Table 3). We found that waist circumference and BMI, two measures of adiposity that were significantly reduced in the fit group, did not significantly correlate with any measure of oxidative stress in our study group. Thus, the effect of fitness on baseline oxidative stress appears to be independent of these two factors. Similarly, HDL-C levels were not correlated with baseline plasma $\mathrm{F}_{2}$-isoprostane levels, but surprisingly there was a weak positive correlation between HDL-C levels and urinary levels of $\mathrm{F}_{2}$-isoprostanes. However, this correlation appears to be driven by gender differences as the women in this study had both significantly higher HDL-C and urinary levels of $\mathrm{F}_{2}$-isoprostane compared to the men. After controlling for gender, HDL-C did not correlate with urinary $\mathrm{F}_{2}$-isoprostane $(P=0.267)$. In any case, the higher levels of HDL-C seen in the fit group clearly cannot account for the decreased level of urinary oxidative stress markers seen in the fit group.

To determine if differences in the levels of oxidative stress biomarkers between fit and unfit individuals could be attributable to small molecular weight antioxidants, we measured two markers of antioxidant status, total antioxidant power (TAP) and reduced glutathione to oxidized glutathione ratio (GSH/GSSG), in baseline plasma samples. TAP did not differ between the groups $(0.61 \pm 0.02$ vs. $0.60 \pm 0.03 \mathrm{mM}$ uric acid equivalents, unfit and fit, respectively, $P>0.05$, Fig. 3a). Although the GSH/GSSG ratio was somewhat higher in fit individuals, this difference was not significant $(14.5 \pm 0.8$ vs. $15.8 \pm 1.0$ in unfit and fit, respectively, $P>0.05$, Fig. $3 b$ ) and GSH/GSSG ratio did not correlate with levels of any oxidative marker (Table 3). These results suggest that differences in baseline levels of oxidative stress biomarkers could not be attributed to significant differences in circulating antioxidants.

Effects of fitness on the response to an acute oxidative insult

Because various other parameters failed to explain the modest decrease in levels of oxidative stress markers in the fit group, we hypothesized that differences might be attributable to an increased ability of fit 
Table 3 Correlation matrix

\begin{tabular}{llllll}
\hline & $\begin{array}{l}\text { I/R response } \\
\text { (AURC) }\end{array}$ & $\begin{array}{l}\text { Plasma F2isoP } \\
\text { (pre) }\end{array}$ & $\begin{array}{l}\text { Urine F2isoP } \\
\text { (sum) }\end{array}$ & 8-oxo-G & 8-OHdG \\
\hline Age & -0.255 & NS & NS & NS & NS \\
BMI & NS & NS & NS & NS & NS \\
WC & NS & NS & NS & NS & NS \\
HLPA score & NS & NS & NS & -0.272 & -0.406 \\
HDL-C & NS & NS & 0.286 & NS & NS \\
TAP & NS & NS & NS & NS & NS \\
GSH/GSSG & -0.364 & NS & NS & NS & NS \\
Plasma F2isoP (pre) & -0.300 & - & NS & NS & 0.244 \\
Urine F2isoP & NS & NS & - & NS & NS \\
8-oxo-G & NS & NS & NS & - & 0.686 \\
8-OHdG & NS & 0.244 & NS & 0.686 & - \\
\hline
\end{tabular}

Significant Pearson correlations shown $(P<0.05)$

$N S$ not significant, $B M I$ body mass index, $W C$ waist circumference, $H L P A$ historical leisure physical activity, TAP total antioxidant power would constantly subject the individual to transient increases in ROS and the resulting upregulation of antioxidant enzymes, priming this response in fit individuals (Radak et al. 2005). In contrast, sedentary older individuals would lack this habitual mild stress and therefore would have a much slower response or possibly lose this response entirely. While priming an antioxidant enzyme response would likely have only modest effects on baseline levels of markers, such a primed response would be expected to have a substantial impact on levels of oxidative markers after an acute, oxidative challenge.

To determine if increased physical fitness improved antioxidant response capacity during an acute oxidative challenge, we measured the changes in levels of plasma $\mathrm{F}_{2}$-isoprostanes in response to moderate forearm $\mathrm{I} / \mathrm{R}$ induced by inflation of a blood pressure cuff. Forearm $\mathrm{I} / \mathrm{R}$ significantly increased $\mathrm{F}_{2}$-isoprostane levels in both groups of subjects $(P<0.001)$. Figure 4 shows the effect of fitness on the capacity to resist oxidative stress as measured by the $\mathrm{F}_{2}$-isoprostane response to the forearm I/R challenge. The fit group had a significantly lower $\mathrm{F}_{2}$-isoprostane response across time (repeated measures) to the $\mathrm{I} / \mathrm{R}$ challenge compared to the unfit group ( $P=0.037$, Fig. 4a). The overall response, calculated as AURC, was significantly lower in the fit group ( $P=0.015$, Fig. $4 \mathrm{~b})$.

Determinants of oxidative stress in response to $\mathrm{I} / \mathrm{R}$ challenge

One antioxidant enzyme that has been hypothesized to play a critical role in response to acute oxidative
Fig. 3 Markers of antioxidant capacity unfit (black bar) and fit (white bar) older adults. a Total antioxidant power. b Reduced to oxidized glutathione ratio (GSH/GSSG). Values are means \pm SEM 

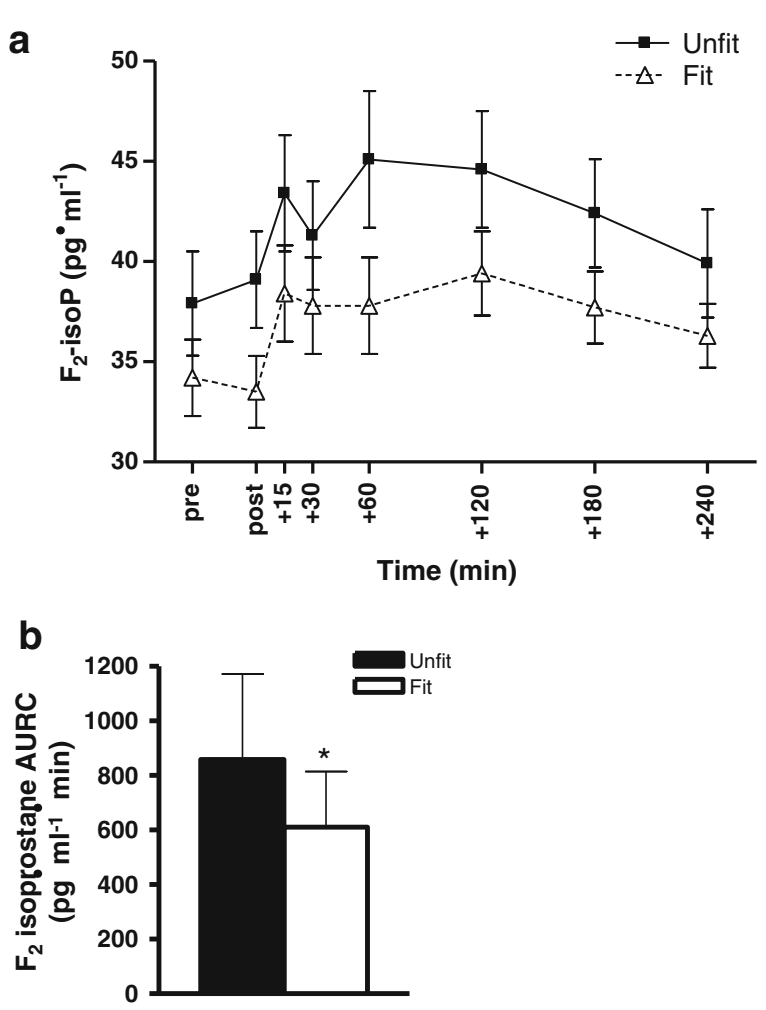

Fig. 4 Plasma $F_{2}$-isoprostane responses to the $I / R$ trial in fit and unfit older adults. Values are means \pm SEM. a Mean levels across time points. Pre to post denotes the time period of the forearm I/R. The fit group (open triangles) had significantly lower $\mathrm{F}_{2}$-isoprostane response to the challenge compared to the unfit (black squares) $(P<0.05)$. b Mean integrated response calculated as AURC for each individual. $* P<0.05$, significantly different from unfit

stress is glutathione peroxidase (GPx). Because this enzyme utilizes glutathione for its activity, individuals with slightly higher GSH/GSSG levels would be expected to have increased GPx activity and lower levels of oxidative stress markers after I/R challenge. When we performed correlation studies to determine if GSH/GSSG ratio correlated with the response to I/R challenge, we found that GSH/GSSG ratio predicted $13 \%$ of the variance in the $\mathrm{F}_{2}$-isoprostane response to the $\mathrm{I} / \mathrm{R}$ trial $(r=-0.364, P=0.002$, Fig. 5). In a regression analysis with $\mathrm{F}_{2}$-isoprostane response (AURC) as the dependent variable, entering GSH/ GSSG, baseline plasma $\mathrm{F}_{2}$-isoprostanes, and age increased the variance explained to $23 \%(r=-0.484$, $P<0.001)$. In this model, age was not a significant predictor.

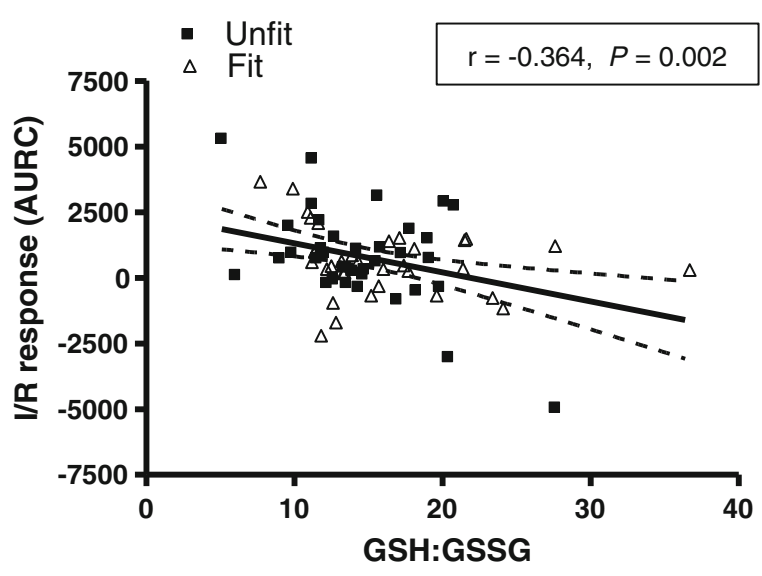

Fig. 5 Pearson correlation between reduced to oxidized glutathione ratios and the $\mathrm{F}_{2}$-isoprostane response to the $\mathrm{I} / \mathrm{R}$ trial (AURC) in all the study participants $(n=72)$. Solid line shows the line of best fit and the dotted lines are $95 \%$ confidence intervals. Fit individuals are denoted by open triangle and unfit individuals by black square

\section{Discussion}

Our results support two significant conclusions in regards to the relationship between physical fitness and oxidative stress. First, that among healthy older adults, increased physical fitness is associated both with modestly lower oxidative stress under resting conditions and substantially lower elevations in oxidative stress after an acute oxidative challenge. Second, the reduced oxidative stress exhibited by the physically fit individuals could not be attributed to known effects of exercise training such as lower adiposity, increased HDL concentrations, or increased plasma concentrations of small molecular weight antioxidants. Therefore, the reduced oxidative stress associated with physical fitness seems most likely to result from differences in activity of antioxidant enzymes.

Previous studies examining the effect of physical fitness on oxidative stress in older adults have been equivocal (Marzatico et al. 1997; Santos-Silva et al. 2001; Pittaluga et al. 2006; Vincent et al. 2006; Knez et al. 2007). Our results suggest that this is because under basal (resting) condition the differences in oxidative stress markers in healthy older individuals are quite modest, and only become readily apparent in response to an oxidative challenge supporting the hypothesis that exercise promotes a greater ability to 
upregulate antioxidant enzymes in response to stressors. Although several previous studies have looked at the effect of exercise training or fitness on the response to an oxidative challenge in older adults, interpreting the results of these previous studies was complicated by the fact that an exercise test was also used as the oxidative challenge (Khassaf et al. 2001; Miyazaki et al. 2001; Sacheck et al. 2003; Fatouros et al. 2004; Choi and Cho 2007; Magalhaes et al. 2007; Nikolaidis et al. 2007; Simar et al. 2007; Falone et al. 2010). Therefore, whether the exercise training increased resistance to oxidative stress in general or specifically to the stress of exercise was unclear. Our use of a novel, non-exercise-related oxidative challenge, forearm $I / R$, suggests that greater fitness is associated with a generalized greater resistance to oxidative stress. This finding is important because most of the stressors associated with oxidative damage and morbidity and mortality in older adults (e.g., infections, physical trauma, and chronic disease) are not related to exercise. Therefore, our finding that fitness correlates with generalized resistance to oxidative stress suggests a plausible mechanism linking reduced morbidity and mortality associated with increased physical fitness. Our results are in agreement with data from animal studies showing that exercise training in older animals results in increased expression and activity of antioxidant enzymes, improved myocardial tolerance to in vivo $\mathrm{I} / \mathrm{R}$, and greater postischemic recovery of ventricular function (Powers et al. 1998; Starnes et al. 2003; Lawler et al. 2009)

Although we did not measure activity levels of antioxidant enzymes in this study, previous studies have shown that exercise training increases expression of antioxidant enzymes including glutathione peroxidase (GPx), superoxide dismutase (SOD), glutathione reductase (GR), and catalase (Khassaf et al. 2001; Miyazaki et al. 2001; Ookawara et al. 2003; Rousseau et al. 2006; Knez et al. 2007). Some investigators have suggested that GPx may be the enzyme most responsive to exercise-induced oxidative stress (Covas et al. 2002; Elosua et al. 2003; Knez et al. 2007). Although there were no significant differences in GSH/GSSG ratios between the fit and unfit group at baseline, we found an inverse relationship between the GSH/GSSG ratio and I/R response. This correlation was stronger in the unfit group compared to the overall cohort, and weaker (and non-significant) in the fit group (data not shown). The reason for these differences is not clear, but if fit individuals have increased GPx and GR activity, they may be able to recycle GSSG rapidly, so they are less affected by changes in overall GSH levels, while individuals with low GPx and GR activity may be more susceptible to damage when low overall GSH levels are present.

A noteworthy aspect of these study results is that the control group of "unfit" individuals was overall quite healthy. This is evidenced by the mean resting blood pressures and the laboratory measures. In actuality, although their fitness levels were lower, these individuals were not necessarily sedentary and reported moderate levels of lifetime physical activity (see HLPA score, Table 2). The fact that a significant difference was nevertheless detected in the markers of oxidative damage and the capacity to neutralize oxidative stress favoring the fit group underscores that physical fitness provides additional resistance from oxidative stress, above and beyond healthy active lifestyle among older adults. We would speculate that even greater differences among older adults might be found when comparing fit individuals to a cohort of truly sedentary individuals or patients with chronic disease. Data on patients with chronic heart failure show that they exhibit lower expression and activity of antioxidant enzymes (SOD, catalase, GPx) in skeletal muscle compared to sedentary age-matched controls (Linke et al. 2005). Along the same lines, diabetic patients exhibit elevated DNA damage compared to non-diabetic patients (Nojima et al. 2008).

An inherent limitation of a cross-sectional study such as ours is that it is difficult to make causal inference and there may be a bias related to selfselection for a more or less active lifestyle. Another potential limitation was the statistically significant difference in age between the fit and the unfit groups. Although one could argue that the clinical relevance of 2.5-year age difference is minimal, this difference was accounted for in all data analyses. The results from this study must be confirmed in a longitudinal design and also to verify that plasticity exists in the redox systems of older sedentary adults. We have already shown that the $\mathrm{F}_{2}$-isoprostane response to the $\mathrm{I} / \mathrm{R}$ trial can be modified using a dietary antioxidant intervention (Traustadóttir et al. 2009); however, the efficacy of an exercise intervention must still be 
investigated. Thus, future interventional studies are required to demonstrate that exercise training of sufficient intensity to increase physical fitness will in fact increase the capacity of older adults to resist oxidative stress. In this regard, we note the findings of a recent 12-month exercise intervention study in overweight, postmenopausal sedentary women (Campbell et al. 2010). Overall, the group that participated in the exercise intervention showed modest increases in fitness and decreases in body weight loss but statistically insignificant decreases in baseline urinary $\mathrm{F}_{2}$-isoprostane levels. However, a planned subgroup analyses showed that women who increased their $\mathrm{VO}_{2}$ max by $>15 \%$ as a result of the exercise training had significant decreases $(14.1 \%)$ in their urinary $\mathrm{F}_{2}$-isoprostane levels (Campbell et al. 2010). Additionally, a small exercise study in older men found that 16 weeks of endurance training reduced levels of the oxidative stress biomarkers (malondialdehyde) and increased GPx activity generated in response to a maximal exercise test (Fatouros et al. 2004). These studies suggest a causal relationship between exercise training and greater resistance to oxidative stress.

The use of sensitive and comprehensive measures to classify physical fitness levels should be noted as a particular strength of the present study. The classification was based on objective measurements of both cardiorespiratory fitness and leg power. $\mathrm{VO}_{2}$ max has been shown to be the best predictor of mortality in men and women (Blair et al. 1996), while leg power has been shown to be the best predictor of functional status and physical performance in older adults (Foldvari et al. 2000; Bean et al. 2002).

Another important question that remains unanswered by our study is whether the modest changes in baseline oxidative stress or the greater resistance to oxidative challenge associated with physical fitness are sufficient to produce significant benefits for the individual in terms of health and disease risk. Although precise studies looking at the effect of exposure to carefully titrated doses of oxidative stress over prolonged periods on health outcomes cannot be performed in humans, several studies point to modest changes in oxidative stress parameters being associated with significant changes in health outcomes. For instance, lower levels of oxidative DNA and RNA damage may indicate diminished risk of cancer (Poulsen et al. 1998; Stoner et al. 2008).
In summary, our results demonstrate that in older adults, greater physical fitness is associated with lower oxidative stress and greater capacity to resist an oxidative challenge. Because this reduction in oxidative stress was not attributed to differences in adiposity, HDL levels, or plasma antioxidants, this suggests that changes in antioxidant enzymes are critical. The clinical implication of these data relate to the idea of optimizing exercise protocols for aging individuals. The ACSM/AHA guidelines for physical activity in older adults specifically address the added health benefits of greater amount of physical activity and greater physical fitness (Nelson et al. 2007). Our data support the concept that encouraging older adults to not just be "physically active" but to be "physically fit" may be an effective strategy to lower oxidative stress and oxidative damage and hence to improve long-term health outcomes.

Acknowledgments We would like to thank Dr. Panayiotis Tsitouras, the KLRI clinical staff, Pat Levin, and Jane Heilman for their invaluable help in the administration and data collection of this study. We also thank our study volunteers for their willingness to participate.

\section{References}

AHA (1972) Exercise testing and training of apparently healthy individuals: a handbook for physicians. American Heart Association, Dallas

American College of Sports Medicine (2005) Guidelines for exercise testing and prescription. Lea \& Febiger, Philadelphia

Ashok BT, Ali R (1999) The aging paradox: free radical theory of aging. Exp Gerontol 34:293-303

Bean J, Kiely DK et al (2002) The relationship between leg power and physical performance in mobility-limited older people. J Am Geriatr Soc 50:461-467

Blair SN, Kampert JB et al (1996) Influences of cardiorespiratory fitness and other precursors on cardiovascular disease and all-cause mortality in men and women. JAMA 276(3):205-210

Bradford MM (1976) A rapid and sensitive method for the quantitation of microgram quantities of protein utilizing the principle of protein-dye binding. Anal Biochem 72:248-254

Brown-Borg HM, Bode AM et al (1999) Antioxidative mechanisms and plasma growth hormone levels: potential relationship in the aging process. Endocrine 11(1):4148

Butterfield DA, Howard B et al (1999) Elevated oxidative stress in models of normal brain aging and Alzheimer's disease. Life Sci 65(18-19):1883-1892 
Campbell PT, Gross MD et al (2010) Effect of exercise on oxidative stress: a 12-month randomized, controlled trial. Med Sci Sports Exerc 42(8):1448-1453

Choi E-Y, Cho Y-O (2007) The effects of physical training on antioxidative status under exercise-induced oxidative stress. Nutr Res Pract 1:14-18

Church TS, Barlow CE et al (2002) Associations between cardiorespiratory fitness and C-reactive protein in men. Arterioscler Thromb Vasc Biol 22(11):1869-1876

Cottreau CM, Ness RB et al (2000) Physical activity and reduced risk of ovarian cancer. Obstet Gynecol 96:609614

Covas MI, Elosua R et al (2002) Relationship between physical activity and oxidative stress biomarkers in women. Med Sci Sports Exerc 34(5):814-819

Davies SS, Traustadottir T et al (2009) Ischemia-reperfusion unveils impaired capacity of older adults to restrain oxidative insult. Free Radic Biol Med 47:1014-1018

de Vos NJ, Singh NA et al (2005) Optimal load for increasing muscle power during explosive resistance training in older adults. J Gerontol A Biol Sci Med Sci 60A(5):638-647

Elosua R, Molina LM et al (2003) Response of oxidative stress biomarkers to a 16-week aerobic physical activity program, and to acute physical activity, in healthy young men and women. Atherosclerosis 167:327-334

Falone S, Mirabilio A et al (2010) Differential impact of acute bout of exercise on redox- and oxidative damage-related profiles between untrained subjects and amateur runners. Physiol Res 59:953-961

Fatouros IG, Jamurtas AZ et al (2004) Oxidative stress responses in older men during endurance training and detraining. Med Sci Sports Exerc 36(12):2065-2072

Foldvari M, Clark M et al (2000) Association of muscle power with functional status in community-dwelling elderly women. J Gerontol Med Sci 55A(4):M192-M199

Griffith OW (1986) Glutathione and glutathione disulfide. In: Bergmeyer HU, Bergmeyer J, Grabi M (eds) Methods of enzymatic analysis. Verlag Chemie, Deerfield Beach, pp 521-529

Harman D (1956) Aging: a theory based on free radical and radiation chemistry. J Gerontol A Biol Sci Med Sci 11:298-300

Harman SM, Liang L et al (2003) Urinary excretion of three nucleic acid oxidation adducts and isoprostane F2-alpha measured by liquid chromatography-mass spectrometry in smokers, ex-smokers, and nonsmokers. Free Radic Biol Med 35(10):1301-1309

Khassaf M, Child RB et al (2001) Time course of responses of human skeletal muscle to oxidative stress induced by damaging exercise. J Appl Physiol 90:1031-1035

Knez WL, Jenkins DG et al (2007) Oxidative stress in half and full Ironman triathletes. Med Sci Sports Exerc 39(2):283288

Kohrt WM, Malley MT et al (1991) Effects of gender, age, and fitness level on response of VO2 max to training in 6071 yr olds. J Appl Physiol 71:2004-2011

Kriska AM (1997) Historical leisure activity questionnaire. Med Sci Sports Exerc 29:S43-S45

Kriska AM, Laporte RE et al (1991) The association of physical activity and diabetic complications in individuals with insulin-dependent diabetes mellitus: the epidemiology of diabetes complications study-VII. J Clin Epidemiol 44:1207-1214

LaMonte MJ, Blair SN et al (2005) Physical activity and diabetes prevention. J Appl Physiol 99(3):1205-1213

Lawler JM, Kwak H-B et al (2009) Exercise training inducibility of MnSOD protein expression and activity is retained while reducing prooxidant signaling in the heart of senescent rats. Am J Physiol 296:R1496-R1502

Liang Y, Wei P et al (2003) Quantification of 8-isoprostaglandin-F2à and 2,3-dinor-8-iso-prostaglandin-F2à in human urine using liquid chromatography-tandem mass spectrometry. Free Radic Biol Med 34(4):409-418

Linke A, Adams V et al (2005) Antioxidative effects of exercise training in patients with chronic heart failure. Circulation 111:1763-1770

Magalhaes J, Ferreira R et al (2007) Indoor climbing elicits plasma oxidative stress. Med Sci Sports Exerc 39(6):955963

Marzatico F, Pansarasa O et al (1997) Blood free radical antioxidant enzymes and lipid peroxides following longdistance and lactacidemic performances in highly trained aerobic and sprint athletes. J Sports Med Phys Fitness 37:235-239

Miyazaki H, Oh-ishi S et al (2001) Strenuous endurance training in humans reduces oxidative stress following exhausting exercise. Eur J Appl Phys 84:1-6

Morrow JD, Roberts LJ 2nd (1999) Mass spectrometric quantification of F2-isoprostanes in biological fluids and tissues as measure of oxidant stress. Methods Enzymol 300:3-12

Nelson ME, Rejeski WJ et al (2007) Physical activity and public health in older adults: recommendation from the American College of Sports Medicine and the American Heart Association. Med Sci Sports Exerc 39(8):14351445

Nikolaidis MG, Paschalis V et al (2007) Decreased blood oxidative stress after repeated muscle damaging exercise. Med Sci Sports Exerc 39(7):1080-1089

Nojima H, Watanabe $\mathrm{H}$ et al (2008) Effect of aerobic exercise training on oxidative stress in patients with type 2 diabetes mellitus. Metab Clin Exp 57(2):170-176

Ookawara T, Haga S et al (2003) Effects of endurance training on three superoxide dismutase isoenzymes in human plasma. Free Radic Res 37(7):713-719

Paffenbarger RS, Hyde RT et al (1993) The association of changes in physical activity level and other lifestyle characteristics with mortality among men. N Engl J Med 328:538-545

Pittaluga M, Parisi P et al (2006) Cellular and biochemical parameters of exercise-induced oxidative stress: relationship with training levels. Free Radic Res 40(6):607-614

Poulsen HE, Prieme H et al (1998) Role of oxidative DNA damage in cancer initiation and promotion. Eur $\mathrm{J}$ Cancer Prev 7(1):9-16

Powers SK, Demirel HA et al (1998) Exercise training improves myocardial tolerance to in vivo ischemiareperfusion in the rat. Am J Physiol 275(5):R1468-R1477

Radak Z, Chung HY et al (2004) Age-associated increases in oxidative stress and nuclear transcription factor $\mathrm{k} B$ activation are attenuated in rat liver by regular exercise. FASEB J 18(6):749-750 
Radak Z, Chung HY et al (2005) Exercise and hormesis: oxidative stress-related adaptation for successful aging. Biogerontology 6:71-75

Rosa EF, Silva AC et al (2005) Habitual exercise program protects murine intestinal, skeletal, and cardiac muscles against aging. J Appl Physiol 99(4):1569-1575

Rousseau AS, Margaritis I et al (2006) Physical activity alters antioxidant status in exercising elderly subjects. J Nutr Biochem 17(7):463-470

Sacheck JM, Milbury PE et al (2003) Effect of vitamin E and eccentric exercise on selected biomarkers of oxidative stress in young and elderly men. Free Radic Biol Med 34 (12):1575-1588

Santos-Silva A, Rebelo MI et al (2001) Leukocyte activation, erythrocyte damage, lipid profile and oxidative stress imposed by high competition physical exercise in adolescents. Clin Chim Acta 306:119-126

Simar D, Malatesta D et al (2007) Physical activity modulates heat shock protein-72 expression and limits oxidative damage accumulation in a healthy elderly population aged 60-90 years. J Gerontol Med Sci 62A(12):14131419

Sohal RS, Weindruch R (1996) Oxidative stress, caloric restriction, and aging. Science 273(5271):59-63

Spector A (2000) Review: oxidative stress and disease. J Ocul Pharmacol Ther 16:193-201
Spina RJ, Rashid S et al (2000) Adaptations in beta-adrenergic cardiovascular responses to training in older women. $\mathrm{J}$ Appl Physiol 89(6):2300-2305

Starnes JW, Taylor RP et al (2003) Exercise improves postischemic function in aging hearts. Am J Physiol Heart Circ Physiol 285(1):H347-H351

Stathokostas L, Jacob-Johnson S et al (2004) Longitudinal changes in aerobic power in older men and women. J Appl Physiol 97(2):781-789

Stoner GD, Wang L-S et al (2008) Laboratory and clinical studies of cancer chemoprevention by antioxidants in berries. Carcinogenesis 29(9):1665-1674

Traustadóttir T, Bosch PR et al (2004) Hypothalamic-pituitaryadrenal axis response and recovery from high-intensity exercise in women: effects of aging and fitness. J Clin Endocrinol Metab 89(7):3248-3254

Traustadóttir T, Stock AA et al (2008) High-dose statin use does not impair aerobic capacity or skeletal muscle function in older adults. Age 30(4):283-291

Traustadóttir T, Davies SS et al (2009) Tart cherry juice decreases oxidative stress in healthy older men and women. J Nutr 139:1896-1900

Vincent HK, Bourguignon C et al (2006) Resistance training lowers exercise-induced oxidative stress and homocysteine levels in overweight and obese older adults. Obesity (Silver Spring) 14(11):1921-1930 